International Journal of Pure and Applied Mathematics

Volume 92 No. 2 2014, 279-296

ISSN: 1311-8080 (printed version); ISSN: 1314-3395 (on-line version)

url: http://www.ijpam.eu

doi: http://dx.doi.org/10.12732/ijpam.v92i2.10

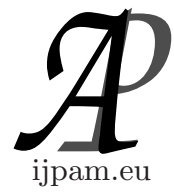

\title{
NONLINEARLY PERTURBED HEAT EQUATION
}

\author{
Maria de Fátima Correia ${ }^{1}$, Carlos Ramos ${ }^{2}$, Sandra Vinagre ${ }^{3 \S}$ \\ ${ }^{1,2,3}$ Department of Mathematics, CIMA-UE \\ University of Évora \\ Rua Romão Ramalho 59, 7000-671, Évora, PORTUGAL
}

\begin{abstract}
We consider the linear heat equation with appropriate boundary conditions describing the temperature on a wire with adiabatic endpoints. We also consider a perturbation, which provokes a global change in the temperature of the wire. This perturbation occurs periodically and is modeled by an iterated nonlinear map of the interval belonging to a one-parameter family of quadratic maps, $f_{\mu}$. We observe a long term stabilization, under time evolution, of the number of new critical points of the temperature function. However, for certain values of the parameter $\mu$, even with the stabilization effect of the number of critical points, the evolution of the temperature function is chaotic. We study the parameters of the system, that is, difusion coeficient and $\mu$, in order to characterize the observed behaviour and its dependence on the topological invariants of $f_{\mu}$, in particular the dependence on the chaotic behaviour of $f_{\mu}$.
\end{abstract}

AMS Subject Classification: 37E05, 39B12, 35K05, 37B40, 37C25, 74H65 Key Words: heat equation, chaotic dynamics, iteration theory, topological entropy

\section{Introduction and Preliminaries}

We consider an ideal system arising from one of the most simple boundary value problem, the linear heat equation describing the temperature on a wire with adiabatic endpoints. When the system is isolated evolves continuously described by the usual linear heat equation and in this regime we have the explicit time dependent solution. Here, we consider that the system is not

Received: February 6, 2014

(C) 2014 Academic Publications, Ltd.

$\S_{\text {Correspondence author }}$ 
isolated and is periodically perturbed. When the perturbation disappears the system evolves again continuously and the process repeats itself after constant time intervals. The considered perturbation is modeled by a nonlinear iterated interval map in a manner described below in detail.

The physical context is here given, considering the temperature function on the ideal wire, simply as a motivation for the study of certain infinite dimensional systems which have, nonetheless, much properties strongly related with iteration of interval maps (one dimensional systems). These infinite dimensional dynamical systems, despite its simple definition, may present very complex behaviour showing some features of real turbulence regarding the balance between coherence versus disorder. In particular, depending on the parameters, the systems present chaotic behaviour, that is, non-periodic evolution and sensitivity to initial conditions, with exponential divergence of two arbitrary close initial temperature functions.

Before giving in detail our systems, we present some preliminaries and discussion related with previous related work.

Consider a $m$-modal map $f$ in the class $C^{1}(I, I)$, for a certain interval $I$, and the class of differentiable functions

$$
\mathcal{A}=\left\{\varphi \in C^{1}([0,1]): \varphi^{\prime}(0)=\varphi^{\prime}(1)=0,|c p(\varphi)|<\infty\right\},
$$

where $|c p(\varphi)|$ denotes the number of critical points of $\varphi$. That is, a function belongs to the class $\mathcal{A}$ if it is differentiable, its derivatives at the endpoints are 0 and its number of critical points are finite. Consider also the operator $T_{f}$ defined by

$$
\begin{aligned}
T_{f}: \mathcal{A} & \rightarrow \mathcal{A} \\
\varphi & \mapsto f \circ \varphi .
\end{aligned}
$$

Note that this operator is well defined since $(f \circ \varphi)^{\prime}(0)=(f \circ \varphi)^{\prime}(1)=0$. Moreover, if $\phi \in \mathcal{A}$ and $\operatorname{Im}(\phi) \subset I$, then $\operatorname{Im}\left(T_{f}^{k} \phi\right) \subset I$, for every $k \in \mathbb{N}$. Therefore, we obtained a discrete infinite dimension dynamical system $\left(\mathcal{A}, T_{f}\right)$ in the sense that we have a set $\mathcal{A}$ (with additional structure, a topology or a metric, for now not specified) and a self map $T_{f}$, which characterizes the discrete time evolution.

In the papers of Severino et al. [1], [2] and [3], Sharkovsky [4] and Vinagre et al. [5] were applied methods and techniques of discrete dynamical systems to study different aspects of the system $\left(\mathcal{A}, T_{f}\right)$, arising from nonlinear boundary value problems associated with the wave equation and a time-delayed Chua's circuit.

In the works of Correia et al. [6] and [7], using techniques of symbolic dynamics, was studied the localization of the critical points and critical values 
of the iterated functions of the class $\mathcal{A}$, by $T_{f}$, for the case in which $T_{f}$ is induced by a $m$-modal map $f$. After, in the papers of Correia et al. [8] and [9] was analyzed the evolution and distribution of the periodic critical values of the iterated functions when the kneading sequences of $f$ are periodic and was studied the growth rate of critical points of the iterated functions comparing it with the growth number of the map $f$. Later, in the work Correia et al. [10] was analyzed the evolution and distribution of the periodic critical values of the iterated functions when the kneading sequences of $f$ are aperiodic.

The particular characteristic of the system $\left(\mathcal{A}, T_{f}\right)$, for certain choices of the map $f$, is that the number of different critical values will always grow with the iteration of $T_{f}$, for almost all initial conditions. This is an essential aspect of the notion of ideal turbulence introduced by Sharkovsky [11] and [12]. Systems, like $\left(\mathcal{A}, T_{f}\right)$ for certain choices of $f$, presents ideal turbulence which the main characteristic is a cascade process which produce structures of arbitrarily small scale, meaning that in the limit the functions, under iteration, become fractal functions or even stochastic functions, depending on the ergodic properties of the map $f$.

Differently with the systems considered in the described papers above, in the systems we work with, in the present paper, the number of critical points does not grow exponentially. On the contrary, it attains a balance between the creation of new critical points, due to the interval map effect, and the destruction of critical points, due to the dissipative effect of the heat equation.

The interval maps we consider in our work, modeling the perturbation, belongs to the well studied quadratic family defined by $f_{\mu}(x)=1-\mu x^{2}$, depending on the parameter $\mu \in(0,2]$, and with $x_{c}=0$ being the unique critical point where $f_{\mu}$ gets the maximal value, see the Figure 1. There is a maximal invariant interval, $[-1,1]$, where the relevant dynamics occurs, that is, the iterates $f_{\mu}^{k}\left(x_{0}\right):=f_{\mu}\left(\ldots f_{\mu}\left(x_{0}\right)\right)$ ( $k$ times) of initial points $x_{0}$ in $[-1,1]$ will belong to $[-1,1]$, for every $k$. For initial points $x_{0}$ outside $[-1,1]$, the iterates $f_{\mu}^{k}\left(x_{0}\right)$, $k \in \mathbb{N}$ diverge to infinity.

We consider the one parameter family of operators $T_{f_{\mu}}$, induced by $f_{\mu}$ on $\mathcal{A}$, defined by

$$
\begin{aligned}
T_{f_{\mu}}: \mathcal{A} & \rightarrow \mathcal{A} \\
\varphi & \mapsto f_{\mu} \circ \varphi .
\end{aligned}
$$

For each parameter $\mu$, the operator $T_{f_{\mu}}$ is well defined since $\left(f_{\mu} \circ \varphi\right)^{\prime}(0)=$ $\left(f_{\mu} \circ \varphi\right)^{\prime}(1)=0$, therefore, we consider the discrete dynamical system $\left(\mathcal{A}, T_{f_{\mu}}\right)$. Since points outside the interval $[-1,1]$ goes to infinity under iteration of $f_{\mu}$, and every iterate of initial points belonging to the interval $[-1,1]$ belongs to $[-1,1]$, the iterates of initial functions $\phi_{0}$ whose image is contained in $[-1,1]$ 


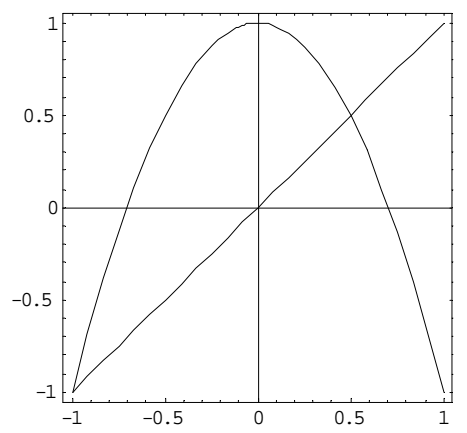

Figure 1: Graph of the quadratic map $f_{\mu}$, with $\mu=2$.

will maintain its image contained in $[-1,1]$. If the image of $\phi_{0}$ is not contained in $[-1,1]$ then the iterates of $\phi_{0}$ under the operator $T_{f_{\mu}}$ will explode. Next, we show an example with several iterates under $T_{f_{\mu}}$ of a given initial function $\phi_{0}$.

Example 1. Consider $f_{\mu}(x)=1-\mu x^{2}$, with $\mu=2$ and

$$
\begin{aligned}
\phi_{0}(x)= & 0.2+0.1 \cos (\pi x)-0.2 \cos (2 \pi x)+0.1 \cos (3 \pi x)+ \\
& +0.1 \cos (4 \pi x)-0.1 \cos (5 \pi x)+0.2 \cos (6 \pi x) .
\end{aligned}
$$

In the Figure 2 we can see increasing frequencies and the asymptotic behaviour expresses the occurrence of ideal turbulence.

\section{Nonlinear Perturbed Heat Equation}

We consider the unit interval representing an ideal wire with isolated endpoints. The temperature function at each point $x \in[0,1]$ and each time instant $t \in \mathbb{R}_{0}^{+}$ is denoted by $\psi(x, t)$. Assume that the wire is such that the time evolution of the temperature function is described by the linear heat equation

$$
\frac{\partial \psi}{\partial t}=\lambda \frac{\partial^{2} \psi}{\partial x^{2}}
$$

where $\lambda$ is a constant, the diffusion coefficient. If there is no heat exchange in the endpoints $x=0$ and $x=1$, we have adiabatic conditions with the correspondent boundary conditions

$$
\frac{\partial \psi}{\partial x}(0, t)=\frac{\partial \psi}{\partial x}(1, t)=0
$$




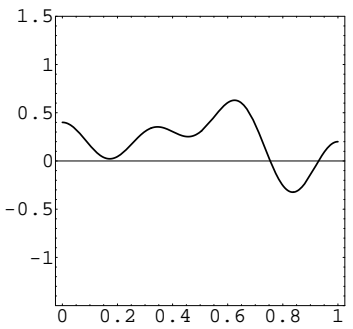

(a)

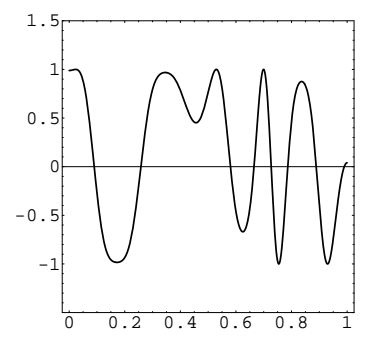

(d)

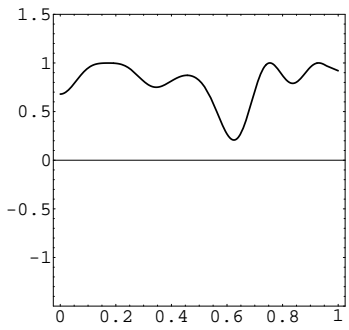

(b)

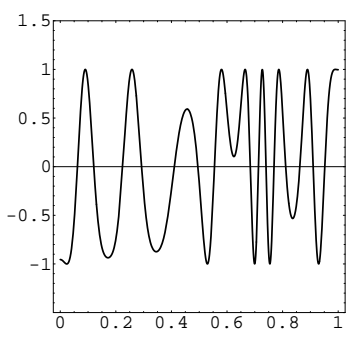

(e)

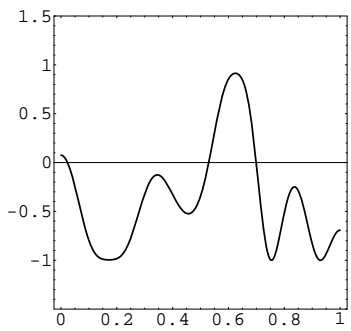

(c)

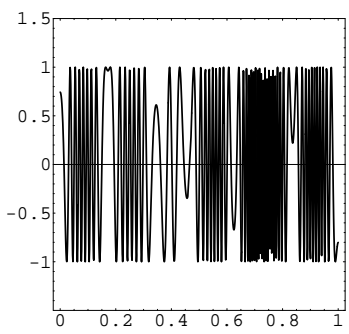

(f)

Figure 2: Graphs of (a) $\phi_{0}(x)$, (b) $\phi_{1}(x)$, (c) $\phi_{2}(x)$, (d) $\phi_{3}(x)$, (e) $\phi_{4}(x)$ and (f) $\phi_{7}(x)$, with $f_{\mu}(x)=1-\mu x^{2}, \mu=2$ and $\phi_{0}(x)=0.2+$ $0.1 \cos (\pi x)-0.2 \cos (2 \pi x)+0.1 \cos (3 \pi x)+0.1 \cos (4 \pi x)-0.1 \cos (5 \pi x)+$ $0.2 \cos (6 \pi x)$.

The initial condition $\psi(x, 0)=\phi_{0}(x)$ is assumed to be a differentiable function belonging to the class $\mathcal{A}$.

The solution can be written as follows

$$
\psi(x, t)=\sum_{n=0}^{\infty} c_{n} e^{-n^{2} \pi^{2} \lambda t} \cos (n \pi x),
$$

where the coefficients $c_{n}$, are determined by the initial condition written as a cosine Fourier series

$$
\phi_{0}(x)=\sum_{n=0}^{\infty} c_{n} \cos (n \pi x) .
$$

Example 2. Let us consider $\lambda=0.005$ and

$$
\begin{aligned}
\psi(x, 0)= & \phi_{0}(x)=0.2+0.1 \cos (\pi x)-0.2 \cos (2 \pi x)+0.1 \cos (3 \pi x)+ \\
& +0.1 \cos (4 \pi x)-0.1 \cos (5 \pi x)+0.2 \cos (6 \pi x) .
\end{aligned}
$$


This initial condition is the same chosen in the Example 1. In Figure 3, we show the evolution of the system in a linear continuous regime for the initial condition $\psi(x, 0)$.

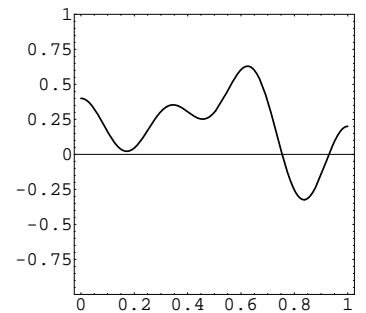

(a)

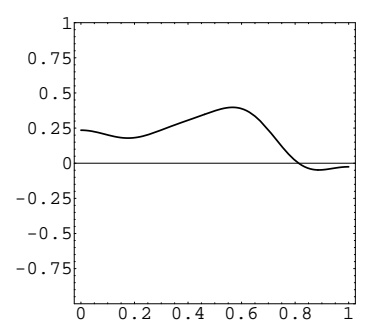

(d)

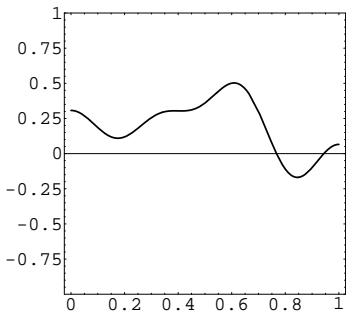

(b)

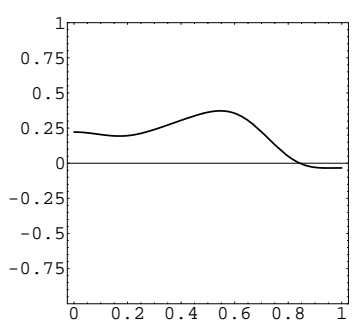

(e)

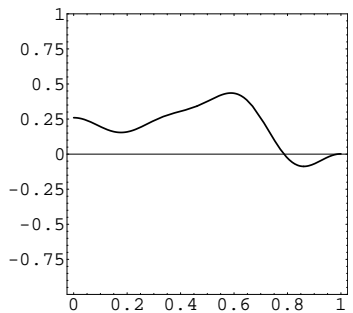

(c)

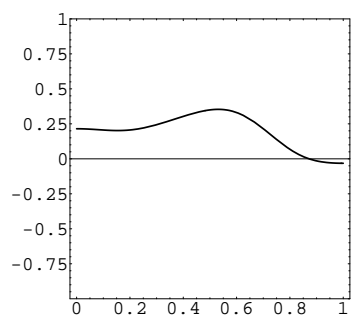

(f)

Figure 3: Graphs of (a) $\psi(x, 0),(\mathrm{b}) \psi(x, 0.4), \quad(\mathrm{c}) \psi(x, 0.8)$, (d) $\psi(x, 1.2),(\mathrm{e}) \psi(x, 1.6)$ and (f) $\psi(x, 2)$, with $\lambda=0.005$ and $\psi(x, 0)=0.2+0.1 \cos (\pi x)-0.2 \cos (2 \pi x)+0.1 \cos (3 \pi x)+0.1 \cos (4 \pi x)-$ $0.1 \cos (5 \pi x)+0.2 \cos (6 \pi x)$.

Now, suppose the system is perturbed in time instants $t_{1}, t_{2}, \ldots$ in a way that the temperature on the wire is globally changed. Being the temperature described initially by the function $\psi_{0}(x, t)$, for $t_{0}<t<t_{1}$, after the perturbation the temperature function is $\psi_{1}(x, t)$, for $t>t_{1}$. We have continuous time evolution for $t \in] t_{j}, t_{j+1}$ [ and discrete time evolution for $t=t_{j}$. We assume that the perturbation is characterized by an nonlinear map $f$ so that $\psi_{j+1}\left(x, t_{j+1}\right)=f\left(\psi_{j}\left(x, t_{j+1}\right)\right)$, with $\psi_{1}\left(x, t_{1}\right)=f\left(\psi_{0}\left(x, t_{1}\right)\right)$. For simplicity and without losing any important property, we choose the time instants to be $t_{k}=k \in \mathbb{N}$. Therefore, the time evolution of the system is described by the sequence of functions

$$
\left\{\psi_{0}, \psi_{1}, \psi_{2}, \ldots, \psi_{k}, \ldots\right\}
$$


each function $\psi_{k}$ satisfying the heat equation for $x \in[0,1], t \in[k, k+1[$ and $k \in \mathbb{N}_{0}$, with initial conditions determined by

$$
\psi_{k+1}(x, k+1)=f\left(\psi_{k}(x, k+1)\right) \text { for } k \in \mathbb{N}_{0},
$$

and $\psi_{0}(x, 0)=\phi_{0}(x)$, a given function from $\mathcal{A}$.

Example 3. Consider $f_{\mu}(x)=1-\mu x^{2}$, with $\mu=2, \lambda=0.00005$ and

$$
\begin{aligned}
\psi_{0}(x, 0)= & 0.2+0.1 \cos (\pi x)-0.2 \cos (2 \pi x)+0.1 \cos (3 \pi x)+ \\
& +0.1 \cos (4 \pi x)-0.1 \cos (5 \pi x)+0.2 \cos (6 \pi x) .
\end{aligned}
$$

In the Figures 4 and 5, we show the evolution of the system described by the heat equation, which is perturbed in time instants $t=1,2,3,8,9,10$. The perturbation changes the system nonlinearly. After the perturbation the system evolves again continuously, and the process repeats itself periodically. Displayed in lines we have the continuous time evolution described by the heat equation in the non-perturbed regime. On the other hand, the first figure in each line is the nonlinear perturbation of the last figure, on the right, in the previous line. In the Figure 4 are presented the temperature function in the time instants $t=0,0.5,1,1,1.5,2,2,2.5,3$ and in the Figure 5 are presented the temperature function in the time instants $t=7,7.5,8,8,8.5,9,9,9.5,10$. In the first Figure, the decay is not perceptible, but, on the contrary, in the second figure, the decay is visible.

Now, we give a formal presentation of the discrete dynamical system used in our work. We again consider the state space $\mathcal{A}$, the operator $T_{f_{\mu}}$, and a new operator $U_{\lambda, \varepsilon}: \mathcal{A} \rightarrow \mathcal{A}$ which gives the time evolution under the unperturbed regime, with diffusion coefficient $\lambda$. The operator $U_{\lambda, \varepsilon}$ is defined implicitly by

$$
U_{\lambda, \varepsilon} \psi(x, t):=\psi(x, t+\varepsilon) .
$$

Remark 1. Note that we could give the operator $U_{\lambda, \varepsilon}$, explicitly and independent of the heat (diffusion) equation. It could be defined in terms of the Fourier and Inverse Fourier transform and an appropriate multiplication operator. For our purposes in the present work this is unnecessary. In future work we will take this approach.

Let us consider the operator $V_{\mu, \lambda, \varepsilon}: \mathcal{A} \rightarrow \mathcal{A}$ defined by

$$
V_{\mu, \lambda, \varepsilon}:=T_{f_{\mu}} \circ U_{\lambda, \varepsilon} .
$$

Now, since the system is periodically perturbed in natural time instants, it is sufficient to consider a natural value for $\varepsilon$. Therefore, we set $\varepsilon=1$, and 


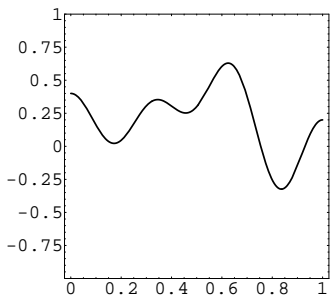

(a)

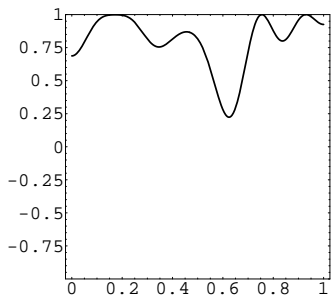

(d)

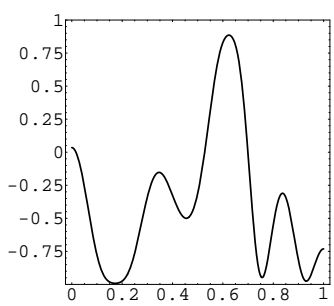

(g)

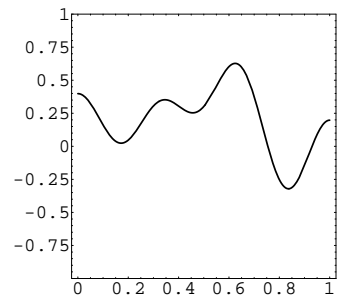

(b)

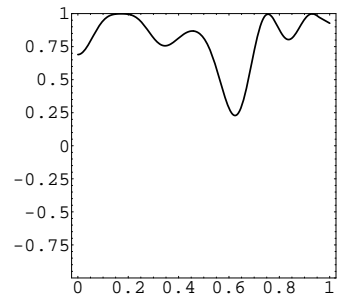

(e)

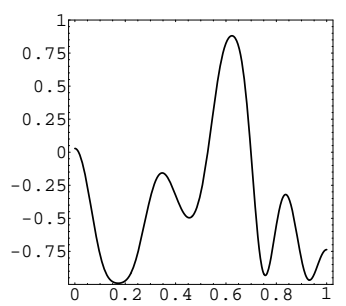

(h)

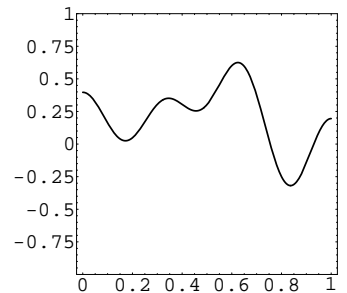

(c)

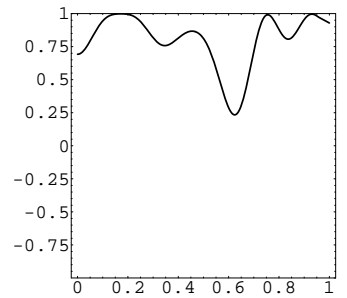

(f)

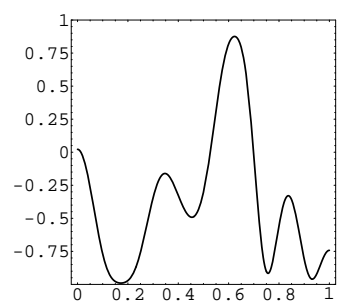

(i)

Figure 4: Graphs of (a) $\psi_{0}(x, 0),(\mathrm{b}) \psi_{0}(x, 0.5),(\mathrm{c}) \psi_{0}(x, 1),(\mathrm{d})$ $\psi_{1}(x, 1),(\mathrm{e}) \psi_{1}(x, 1.5)$, (f) $\psi_{1}(x, 2)$, (g) $\psi_{2}(x, 2)$, (h) $\psi_{2}(x, 2.5)$ and (i) $\psi_{2}(x, 3)$, with $\lambda=0.00005, f_{\mu}(x)=1-\mu x^{2}, \mu=2$ and $\psi_{0}(x, 0)=0.2+0.1 \cos (\pi x)-0.2 \cos (2 \pi x)+0.1 \cos (3 \pi x)+0.1 \cos (4 \pi x)-$ $0.1 \cos (5 \pi x)+0.2 \cos (6 \pi x)$. 


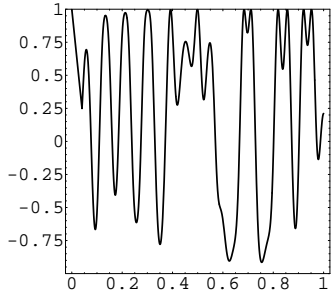

(a)

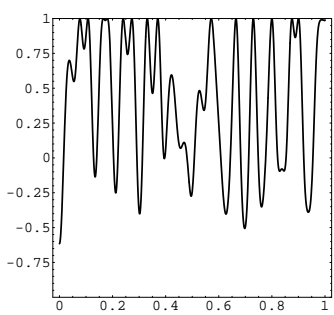

(d)

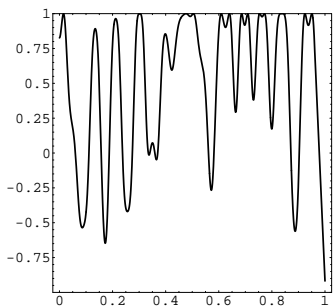

(g)

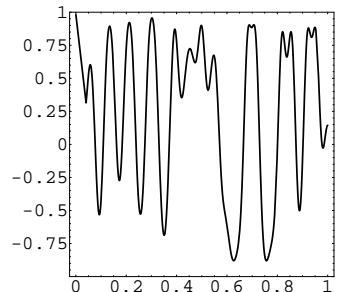

(b)

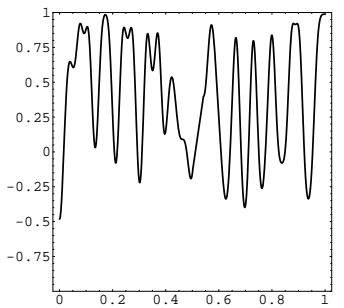

(e)

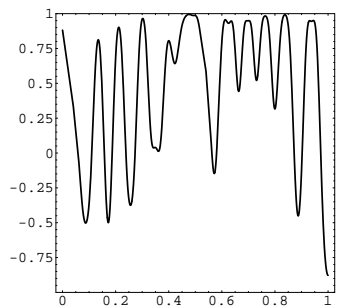

(h)

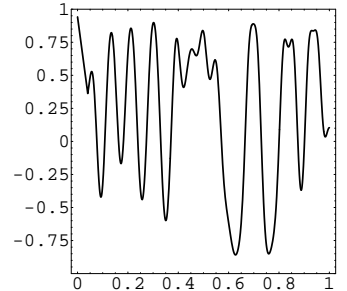

(c)

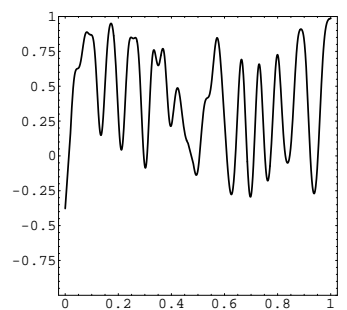

(f)

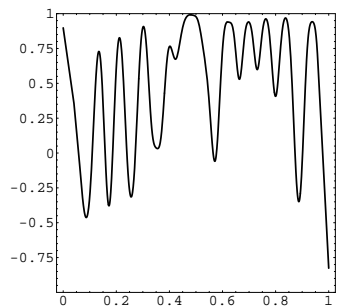

(i)

Figure 5: Graphs of (a) $\psi_{7}(x, 7)$, (b) $\psi_{7}(x, 7.5)$, (c) $\psi_{7}(x, 8)$, (d) $\psi_{8}(x, 8),(\mathrm{e}) \psi_{8}(x, 8.5)$, (f) $\psi_{8}(x, 9),(\mathrm{g}) \psi_{9}(x, 9),(\mathrm{h}) \psi_{9}(x, 9.5)$ and (i) $\psi_{9}(x, 10)$, with $\lambda=0.00005, f_{\mu}(x)=1-\mu x^{2}, \mu=2$ and $\psi_{0}(x, 0)=0.2+0.1 \cos (\pi x)-0.2 \cos (2 \pi x)+0.1 \cos (3 \pi x)+0.1 \cos (4 \pi x)-$ $0.1 \cos (5 \pi x)+0.2 \cos (6 \pi x)$. 
we define $V_{\mu, \lambda} \equiv V_{\mu, \lambda, 1}$. Our discrete dynamical system is, then, defined by the pair $\left(\mathcal{A}, V_{\mu, \lambda}\right)$. When we iterate a function $\phi_{0}(x)$ in $\mathcal{A}$, under $V_{\mu, \lambda}$, the obtained iterates $\phi_{k}(x)=V_{\mu, \lambda}^{k}\left(\phi_{0}(x)\right)$ will correspond to the solution given by the sequence of functions (5) in the time instants $\phi_{k}(x)=\psi_{k}(x, k)$. If, for some reason, we need to obtain the temperature function at a non integer time instant $t^{\prime}$ we simply use the solution presented in (3) with initial condition given by $\psi(x, 0)=V_{\mu, \lambda}^{k}\left(\phi_{0}(x)\right)$, where $k=\left[t^{\prime}\right]$ the integer part of $t^{\prime}$. Then, we evaluate the function for the time instant $t^{\prime}-k$, that is, $\psi\left(x, t^{\prime}-k\right)$.

\section{Stabilization of the Number of Critical Points for Positive Topological Entropy of $f_{\mu}$}

The topological entropy, $h_{t}(g)$, of an interval map, $g$, is an important measure for the characterization of the complex behaviour of the map under iteration, see for details [13]. Roughly speaking as greater topological entropy more complex is the dynamical behaviour. Among other aspects, the topological entropy measures the growth rate of the periodic orbits for $g$. For the infinite dimensional system $\left(\mathcal{A}, T_{g}\right)$, the topological entropy of $g$ measures the growth rate of the number of critical points for the functions in $\mathcal{A}$. That is, if the topological entropy is 0 , then the growth rate is polynomial or there is no growth at all, for almost every initial functions. For positive topological entropy the iterates will have increasingly number of critical points, growing exponentially under iteration. In this case, since the domain of the functions is an interval, in the limit the iterates will tend to a fractal function, and this is the essence of the ideal turbulence.

For the dynamical system $\left(\mathcal{A}, V_{\mu, \lambda}\right)$, independently on the initial condition, once fixed the diffusion coefficient and the parameter $\mu$ so that $f_{\mu}$ has positive topological entropy, we observe that the number of new critical points, in the time evolution of the temperature function, tend to stabilize. This deeply contrasts with the the purely iteration of the operator $T_{f_{\mu}}$.

In Figure 6, we show the evolution of the number of critical points, $N_{k}$, of $\psi(x, t)$, with $t=0,0.2,0.4, \ldots, 1.8,2$, for six different initial conditions.

It is clear that independently of the number of critical points of the initial condition $\psi_{0}$ the iterates $\psi_{k}$ will have the number of critical points stabilized. Since the rate of appearance of new critical points for the iterates is constant and equal to the growth number of the interval map $f_{\mu}$ (therefore is equal to $\left.e^{h_{t}\left(f_{\mu}\right)}\right)$ the rate of destruction is not constant under iteration. It depends on the number of critical points of the temperature function, that is, if the temperature 


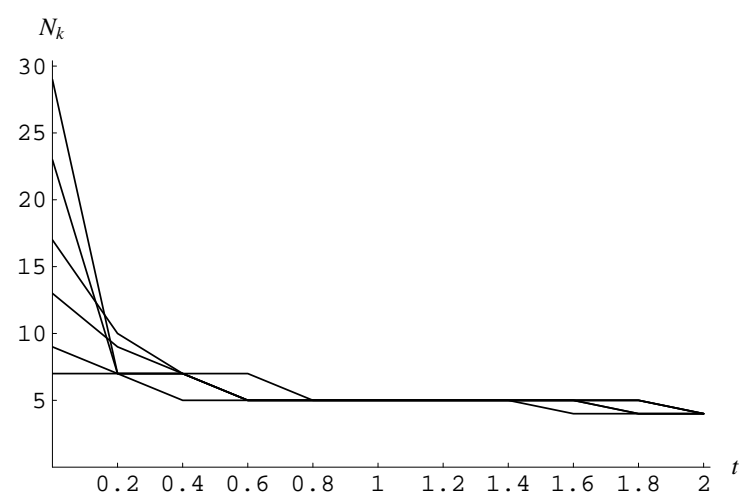

Figure 6: Variation of the number of critical points of $\psi(x, t)$, with $t=$ $0,0.2,0.4, \ldots, 1.8,2$, and $\lambda=0.005$, for six different initial conditions.

function has many critical points the destruction rate will be higher than the destruction rate for an initial temperature function with few critical points. This can be seen in the Figure 6 where the six initial temperature functions have, respectively, 7, 9, 13, 17, 23, and 29 critical points and after 0.4 time units the temperature functions have around 6 critical points.

Example 4. Consider $f_{\mu}(x)=1-\mu x^{2}$, with $\mu=2$,

$$
\begin{aligned}
\psi_{0}(x, 0)= & 0.2+0.1 \cos (\pi x)-0.2 \cos (2 \pi x)+0.1 \cos (3 \pi x)+ \\
& +0.1 \cos (4 \pi x)-0.1 \cos (5 \pi x)+0.2 \cos (6 \pi x)
\end{aligned}
$$

and

$$
\widetilde{\psi}_{0}(x, 0)=0.2+0.1 \cos (\pi x)-0.2 \cos (2 \pi x)+0.1 \cos (3 \pi x) .
$$

In the Figure 7, we show the evolution of the number of critical points, $N_{k}$, of the iterated functions $V_{\mu, \lambda}^{k}\left(\psi_{0}\right)$ and $V_{\mu, \lambda}^{k}\left(\widetilde{\psi}_{0}\right)$, with $k=5, \ldots, 100$, when we fix $\lambda=0.00005$. In the Figure 8, we show the evolution of the number of critical of $V_{\mu, \lambda}^{60}\left(\psi_{0}\right)$ and $V_{\mu, \lambda}^{60}\left(\widetilde{\psi}_{0}\right)$ for different values of the diffusion coefficient $\lambda$.

With our numerical study, we are led to the following result.

Numerical Result 1. Independently of the topological entropy of $f_{\mu}$, that is, independently of $\mu$, of the diffusion coefficient $\lambda$ and of the initial condition, there exists an order after which the number of critical points maintains limited.

Remark 2. i) The average number of critical points of the iterates depends on the diffusion coefficient $\lambda$. When we decrease the diffusion coefficient $\lambda$, the 


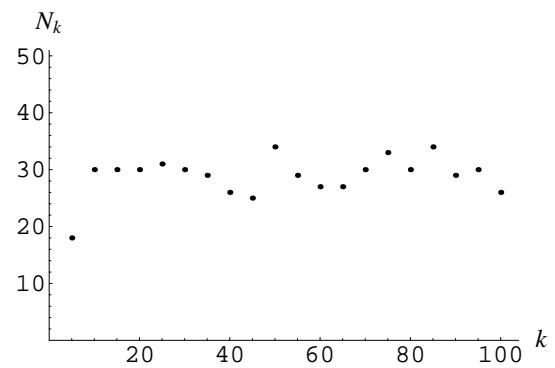

(a)

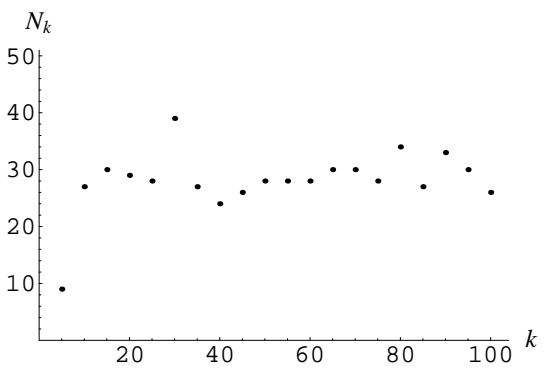

(b)

Figure 7: Variation of the number of critical points of (a) $V_{\mu, \lambda}^{k}\left(\psi_{0}\right)$ and (b) $V_{\mu, \lambda}^{k}\left(\widetilde{\psi}_{0}\right)$, with $k=5, \ldots, 100, \lambda=0.00005, f_{\mu}(x)=$ $1-\mu x^{2}, \mu=2, \psi_{0}(x, 0)=0.2+0.1 \cos (\pi x)-0.2 \cos (2 \pi x)+$ $0.1 \cos (3 \pi x)+0.1 \cos (4 \pi x)-0.1 \cos (5 \pi x)+0.2 \cos (6 \pi x)$ and $\widetilde{\psi}_{0}(x, 0)=$ $0.2+0.1 \cos (\pi x)-0.2 \cos (2 \pi x)+0.1 \cos (3 \pi x)$.

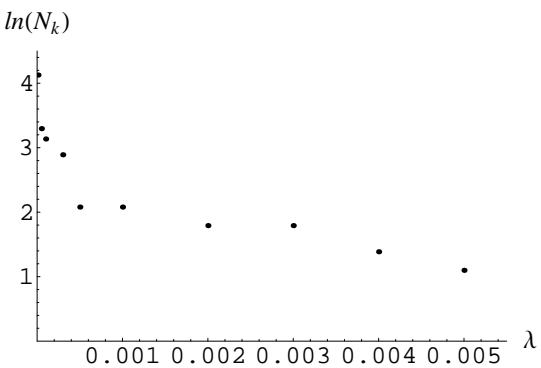

(a)

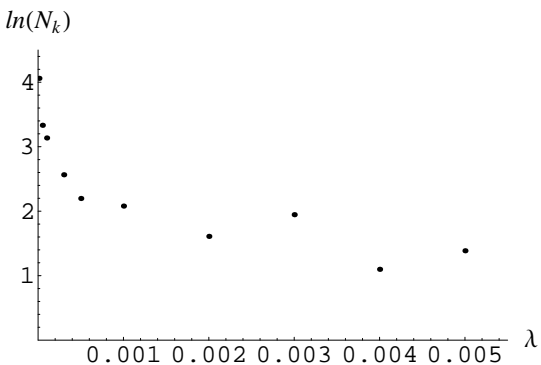

(b)

Figure 8: Variation of the number of critical points of (a) $V_{\mu, \lambda}^{60}\left(\psi_{0}\right)$ and (b) $V_{\mu, \lambda}^{60}\left(\widetilde{\psi_{0}}\right)$, for different values of $\lambda, f_{\mu}(x)=1-\mu x^{2}$, $\mu=2, \psi_{0}(x, 0)=0.2+0.1 \cos (\pi x)-0.2 \cos (2 \pi x)+0.1 \cos (3 \pi x)+$ $0.1 \cos (4 \pi x)-0.1 \cos (5 \pi x)+0.2 \cos (6 \pi x)$ and $\widetilde{\psi}_{0}(x, 0)=0.2+$ $0.1 \cos (\pi x)-0.2 \cos (2 \pi x)+0.1 \cos (3 \pi x)$. 
average number of critical points, at which the temperature function stabilizes, increases, see Table 1.

ii) When we have positive topological entropy (chaos) for the nonlinear perturbation, for low values of the diffusion coefficient, we have a balance between creation and destruction of critical points.

\begin{tabular}{|c|c|c|c|c|c|c|}
\hline \multirow{2}{*}{$\begin{array}{c}\text { intial } \\
\text { condition }\end{array}$} & \multicolumn{2}{|c|}{$\lambda=0.0001$} & \multicolumn{2}{c|}{$\lambda=0.00005$} & \multicolumn{2}{c|}{$\lambda=0.00001$} \\
\cline { 2 - 7 } & $\begin{array}{c}\text { arithmetic } \\
\text { mean }\end{array}$ & $\begin{array}{c}\text { standard } \\
\text { deviation }\end{array}$ & $\begin{array}{c}\text { arithmetic } \\
\text { mean }\end{array}$ & $\begin{array}{c}\text { standard } \\
\text { deviation }\end{array}$ & $\begin{array}{c}\text { arithmetic } \\
\text { mean }\end{array}$ & $\begin{array}{c}\text { standard } \\
\text { deviation }\end{array}$ \\
\hline$\psi_{0}$ & 21,16 & 2,39 & 29,05 & 3,28 & 61,42 & 5,45 \\
\hline$\Psi_{0}$ & 20,32 & 2,58 & 30,00 & 3,20 & 64,26 & 4,24 \\
\hline$\varphi_{0}$ & 21,11 & 1,97 & 29,47 & 2,46 & 64,37 & 4,13 \\
\hline $\bar{\varphi}_{0}$ & 20,05 & 2,48 & 28,26 & 2,88 & 60,53 & 6,65 \\
\hline$\alpha_{0}$ & 21,47 & 2,30 & 29,05 & 3,52 & 62,79 & 4,05 \\
\hline
\end{tabular}

Table 1: The arithmetic mean and the standard deviation for five different initial conditions, $\psi_{0}, \widetilde{\psi}_{0}, \varphi_{0}, \widetilde{\varphi}_{0}, \alpha_{0}$, of the number of critical points. The values are presented for three different values of the diffusion coefficient, $\lambda$.

\section{Behaviour for Topological Entropy Equal to 0}

For the cases in which the quadratic map $f_{\mu}$ has entropy equal to zero every point in the interval, under iteration of $f_{\mu}$, will be attracted to the critical orbit which will have period $2^{k}$ for a certain natural $k$. Moreover, the repulsive coexisting periodic orbits are of period $2^{j}$ with $j<k$. This is verified for values of the parameter $\mu$ below the Feigenbaum point $\mu_{2^{\infty}}$ for which the map $f_{\mu}$ has a nonperiodic critical orbit, has zero topological entropy and has coexisting repulsive periodic orbits of period $2^{j}$, for every natural $j$, see [13] for more details.

A natural question, given a certain dynamical system, in particular the dynamical system defined in our work, is wether there are periodic orbits. In our context, a periodic orbit, will be a sequence of functions $\left\{\psi_{0}, \psi_{1}, \ldots, \psi_{k}, \psi_{0}, \ldots\right\}$, so that $V_{\mu, \lambda}^{k}\left(\psi_{0}\right)=\psi_{0}$, for a certain $k$. It is natural to expect that in the zero entropy case we could have periodic orbits, since every point under iteration of $f_{\mu}$ converges to the critical periodic orbit. The unexpected phenomena is that, once fixed the parameters so that $f_{\mu}$ has zero entropy and a periodic critical orbit of period $2^{k}$, every initial condition $\psi_{0}$, under iteration, converges to a different periodic orbit. This means that the number of distinct periodic orbits, of period $2^{k}$, is infinite. In the following example we show two distinct attracting periodic orbits of period 4 . 
Example 5. Consider $f_{\mu}(x)=1-\mu x^{2}$, with $\mu=1.3107 \ldots, \lambda=0.00005$,

$$
\begin{aligned}
\psi_{0}(x, 0)= & 0.2+0.1 \cos (\pi x)-0.2 \cos (2 \pi x)+0.1 \cos (3 \pi x)+ \\
& +0.1 \cos (4 \pi x)-0.1 \cos (5 \pi x)+0.2 \cos (6 \pi x)
\end{aligned}
$$

and

$$
\begin{aligned}
\widetilde{\psi}_{0}(x, 0)= & 0.2+0.1 \cos (\pi x)-0.2 \cos (2 \pi x)+0.1 \cos (3 \pi x)+ \\
& +0.1 \cos (4 \pi x)-0.1 \cos (5 \pi x)+0.2 \cos (6 \pi x)+ \\
& +0.1 \cos (7 \pi x)+0.2 \cos (8 \pi x)+0.2 \cos (9 \pi x) .
\end{aligned}
$$

In the Figure 9, we present the graphs of $V_{\mu, \lambda}^{k}\left(\psi_{0}\right)$, with $k=95, \ldots, 100$, and, in the Figure 10, we present the graphs of $V_{\mu, \lambda}^{k}\left(\widetilde{\psi}_{0}\right)$, with $k=95, \ldots, 100$, when the critical point of $f_{\mu}$ is a periodic point with period 4 .

For the considered parameter $\mu$ the topological entropy of $f_{\mu}$ is equal to zero, and for $k$ large enough the system approach a periodical function.

We can, after a systematic analysis, state the following

Numerical Result 2. Let $f_{\mu}$ be a quadratic map, such that $\mu<\mu_{2}$, $\psi_{0} \in \mathcal{A}$ and $\psi_{k}=V_{\mu, \lambda}^{k}\left(\psi_{0}\right)$, with $k \in \mathbb{N}_{0}$. Then,

$$
\lim _{n}\left|V_{\mu, \lambda}^{n+2^{m}}\left(\psi_{0}\right)-V_{\mu, \lambda}^{n}\left(\psi_{0}\right)\right|=0
$$

for a certain $m \in \mathbb{N}$.

\section{Conclusions}

Independently of the physical motivation with a wire or the heat equation, the heat equation may be substituted by an abstract diffusion equation. The important point is that the state space is a function space, $\mathcal{A}$, easy to define and the dynamics is given by the iteration of a simple operator $V_{\mu, \lambda}$. This setting generalizes the traditional iteration of interval maps, since the constant functions belongs to the class $\mathcal{A}$. In fact, if the initial function is constant the iteration of $V_{\mu, \lambda}$ reduces to the iteration of the interval map $f_{\mu}$. The generalization is to an infinite dimensional system: a point is substituted by a function (or an infinite vector) and the iteration of a map is substituted by the iteration of an operator.

The main result, in our work, is that, in certain conditions - namely positive topological entropy of $f_{\mu}$ - the iterates, under $V_{\mu, \lambda}$, have an exponential grow of 


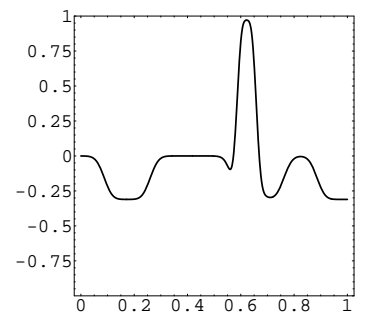

(a)

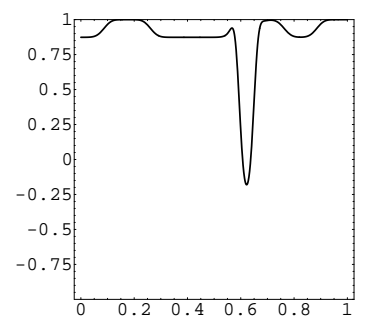

(d)

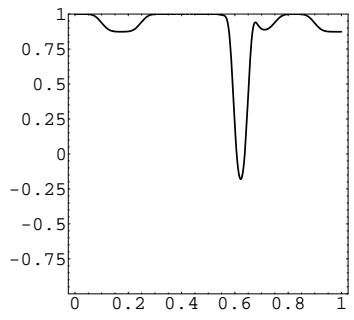

(b)

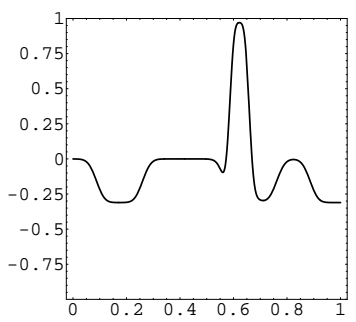

(e)

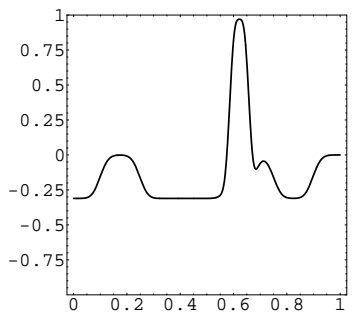

(c)

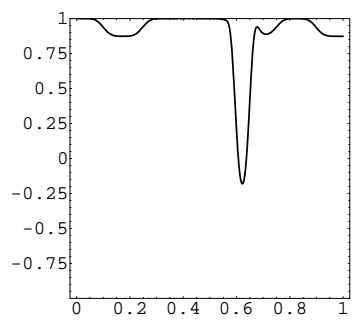

(f)

Figure 9: Graphs of (a) $V_{\mu, \lambda}^{95}\left(\psi_{0}\right), \quad$ (b) $V_{\mu, \lambda}^{96}\left(\psi_{0}\right), \quad$ (c) $V_{\mu, \lambda}^{97}\left(\psi_{0}\right)$, (d) $V_{\mu, \lambda}^{98}\left(\psi_{0}\right)$, (e) $V_{\mu, \lambda}^{99}\left(\psi_{0}\right)$ and (f) $V_{\mu, \lambda}^{100}\left(\psi_{0}\right)$, with $\lambda=0.00005$, $f_{\mu}(x)=1-\mu x^{2}, \mu=1.3107 \ldots, \psi_{0}(x, 0)=0.2+0.1 \cos (\pi x)-$ $0.2 \cos (2 \pi x)+0.1 \cos (3 \pi x)+0.1 \cos (4 \pi x)-0.1 \cos (5 \pi x)+0.2 \cos (6 \pi x)$ and $\widetilde{\psi}_{0}(x, 0)=0.2+0.1 \cos (\pi x)-0.2 \cos (2 \pi x)+0.1 \cos (3 \pi x)+$ $0.1 \cos (4 \pi x)-0.1 \cos (5 \pi x)+0.2 \cos (6 \pi x)+0.1 \cos (7 \pi x)+0.2 \cos (8 \pi x)+$ $0.2 \cos (9 \pi x)$. 


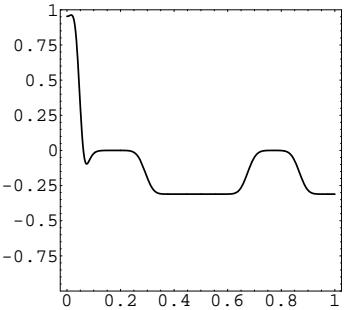

(a)

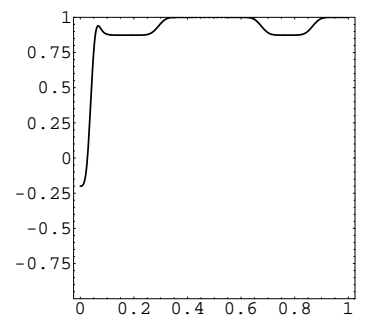

(d)

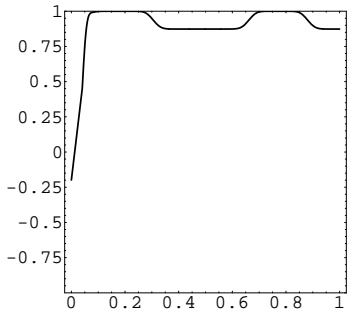

(b)

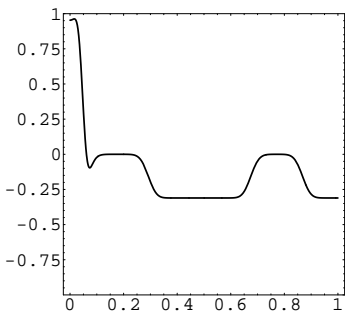

(e)

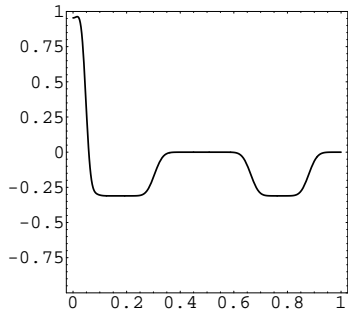

(c)

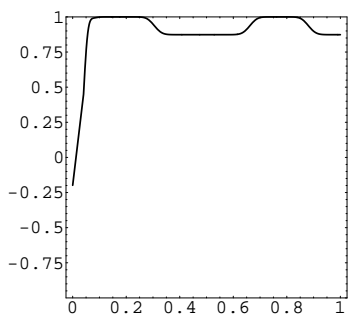

(f)

Figure 10: Graphs of (a) $V_{\mu}{ }^{95}\left(\widetilde{\psi}_{0}\right), \quad$ (b) $V_{\mu}{ }^{96}\left(\widetilde{\psi}_{0}\right), \quad$ (c) $V_{\mu, \lambda}^{97}\left(\widetilde{\psi}_{0}\right)$, (d) $V_{\mu, \lambda}^{98}\left(\widetilde{\psi}_{0}\right)$, (e) $V_{\mu, \lambda}^{99}\left(\widetilde{\psi}_{0}\right)$ and (f) $V_{\mu, \lambda}{ }^{100}\left(\widetilde{\psi}_{0}\right)$, with $\lambda=0.00005$, $f_{\mu}(x)=1-\mu x^{2}, \mu=1.3107 \ldots, \psi_{0}(x, 0)=0.2+0.1 \cos (\pi x)-$ $0.2 \cos (2 \pi x)+0.1 \cos (3 \pi x)+0.1 \cos (4 \pi x)-0.1 \cos (5 \pi x)+0.2 \cos (6 \pi x)$ and $\widetilde{\psi}_{0}(x, 0)=0.2+0.1 \cos (\pi x)-0.2 \cos (2 \pi x)+0.1 \cos (3 \pi x)+$ $0.1 \cos (4 \pi x)-0.1 \cos (5 \pi x)+0.2 \cos (6 \pi x)+0.1 \cos (7 \pi x)+0.2 \cos (8 \pi x)+$ $0.2 \cos (9 \pi x)$. 
number of critical points up to a certain level. After attaining a certain number of critical points, which depends on the parameters, this number oscillates and becomes limited, as we can see in the Figure 7 and in the Table 1. The existence and the dependence of this threshold level for the number of critical points on the parameters can be seen in the Figures 6 and 8 .

The other result, we obtained, which turned out to be unexpected, occurs for the case the parameter $\mu$ is below the Feigenbaum point. In this case every point, under iteration of $f_{\mu}$, is attracted to a periodic orbit of period $2^{j}$, for a certain natural number $j$, and we observe the existence of infinite periodic orbits for the iteration of the operator $V_{\mu, \lambda}$. Each initial condition is, in fact, attracted to a different periodic orbit of period $2^{j}$.

\section{Acknowledgements}

This work has been partially supported by the research center CIMA-UE, FCTPortugal funding program.

\section{References}

[1] R. Severino, A. N. Sharkovsky, J. Sousa Ramos, S. Vinagre, Symbolic Dynamics in Boundary Value problems, Grazer Math. Ber, 346 (2004), 393-402.

[2] R. Severino, A. N. Sharkovsky, J. Sousa Ramos, S. Vinagre, Topological invariants in a model of a time-delayed Chua's circuit, Nonlinear Dynamics, 44 (2006), 81-90. (doi: 10.1007/s11071-006-1942-4)

[3] R. Severino, A. N. Sharkovsky, J. Sousa Ramos, S. Vinagre, Computing topological invariants in boundary value problems reducible to difference equations, Proceedings of the International Conference on Difference Equations, Special Functions and Orthogonal Polynomials, World Scientific Publishing, (2007), 741-751. (doi: 10.1142/9789812770752_0060)

[4] A. N. Sharkovsky, R. Severino, S. Vinagre, Difference Equations and Nonlinear Boundary Value Problems for Hyperbolic Systems, Discrete Dynamics and Difference Equations - Proceedings of the Twelfth International Conference on Difference Equations and Applications, World Scientific Publishing, (2010), 400-409. (doi: 10.1142/9789814287654_0034) 
[5] S. Vinagre, R. Severino, J. Sousa Ramos, Topological invariants in nonlinear boundary value problems, Chaos Solitons Fractals, 25 (2005), 65-78. (doi: 10.1016/j.chaos.2004.11.043)

[6] M. F. Correia, C. C. Ramos, S. Vinagre, Symbolic dynamics for iterated smooth maps, Grazer Math. Ber., 354 (2009), 26-36.

[7] M. F. Correia, C. C. Ramos, S. Vinagre, On the iteration of smooth maps, Discrete Dynamics and Difference Equations - Proceedings of the Twelfth International Conference on Difference Equations and Applications, World Scientific Publishing, (2010), 223-230. (doi: 10.1142/9789814287654_0016)

[8] M. F. Correia, C. C. Ramos, S. Vinagre, Iteration of differentiable functions under m-modal maps, Int. J. of Pure and Applied Math., 70 (2011), 151166.

[9] M. F. Correia, C. C. Ramos, S. Vinagre, Iteration of differentiable functions under m-modal maps with aperiodic kneading sequences, International Journal of Mathematics and Mathematical Sciences, (2012), 17 pages. (doi: $10.1155 / 2012 / 796180)$

[10] M. F. Correia, C. C. Ramos, S. Vinagre, The evolution and distribution of the periodic critical values of iterated differentiable functions, Nonlinear Analysis, 75 (2012), 6343-6359. (doi: 10.1016/j.na.2012.07.011)

[11] A. N. Sharkovsky, Ideal Turbulence, Nonlinear Dynamics, 44 (2006), 1527. (doi: 10.1007/s11071-006-1931-7)

[12] A. N. Sharkovsky, Ideal turbulence and problems of its visualization, Proceedings of the International Conference on Difference Equations, Special Functions and Orthogonal Polynomials, World Scientific Publishing, (2007), 617-635. (doi: 10.1142/S0218127494000216)

[13] J. Milnor, W. Thurston, On Iterated Maps of the Interval, Proceedings Univ. Maryland (ed. J. C. Alexander), 1986-1987, Lect. Notes in Math., 1342, Springer-Verlag, Berlin, New York (1988), 465-563. (doi: 10.1007/BFb0082847) 